\title{
ЕСТЕСТВЕННОЕ ТЕЧЕНИЕ
}

\section{ВРОЖАЕННОЙ АЕФОРМАЦИИ ПОЗВОНОЧНИКА

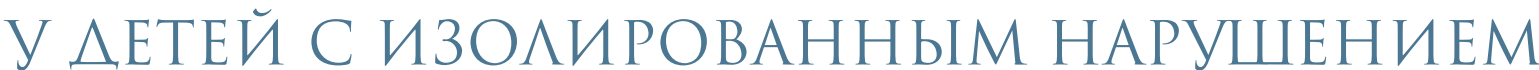 ФОРМИРОВАНИЯ ПОЗВОНКА В ПОЯСНИЧНОМ ОТАЕАЕ}

\author{
С.В. Виссарионов ${ }^{1,2}$, К.А. Картавенко 1 , А.Н. Кокуиин ${ }^{1}$ \\ ${ }^{1}$ Научно-исследовательский детскии́ ортопедический институт им. Г.И. Турнера, Санкт-Петербург, Россия \\ ${ }^{2}$ Северо-Западныи́ государственныи́ медииинскии́ университет им. И.И. Мечникова, Санкт-Петербург, Россия
}

Цель исследования. Оценка характера естественного течения деформации поясничного отдела позвоночника у детей с изолированным нарушением формирования позвонка.

Материал и методы. Проанализированы результаты есте ственного течения деформации позвоночника при нарушении формирования позвонка в поясничном отделе у 40 пациентов в возрасте от 1 года 7 мес. до 17 лет 11 мес., которые получали консервативное лечение и динамическое наблюдение на протя жении четырех лет. Спондилографию проводили в прямой и бо ковой проекциях с кратностью один раз в 6 мес. в положении пациента лежа. Оценивали величину угла локального сколиоти ческого и кифотического компонентов деформации в процес се роста ребенка и величину угла общего поясничного лордоза. Результаты. Для двух компонентов деформации (сколиоза, кифоза) характерно прогрессирующее и стабильное течение. На фоне имеющегося локального искривления в сагиттальной плоскости результаты исследования общего лордоза распределялись в соответствии с величиной возрастной нормы и с уплощением относительно нормы на момент последнего наблюдения. Заключение. При первичном осмотре пациента прогностически ведущую роль в определении дальнейшего характера течения деформации играет величина исходной сколиотической дуги по Cobb. При значении основной дуги деформации меньше $30^{\circ}$ на фоне консервативной терапии отмечается стабильное течение врожденного искривления, а у некоторых детей и тенденция к самокоррекции. У детей с исходной величиной основной сколиотической дуги более $30^{\circ}$ неуклонно прогрессирует врожденная деформация поясничного отдела позвоночника (больше $6^{\circ}$ за 4 года), что является показанием к хирургическому лечению уже в раннем возрасте.

Ключевые слова: поясничный отдел позвоночника, врожденный сколиоз, полупозвонок, стабильная врожденная деформация, прогрессирующая врожденная деформация.

Аля цитирования: Виссарионов С.В., Картавенко К.А., Кокушин А.Н. Естественное течение врожденной десормации позвоночника у детеи́ с изолированным нарушением формирования позвонка в поясничном отделе // Хирургия позвоночника. 2018. Т. 15. № 1. С. 6-17.

DOI: http://dx.doi.org/10.14531/ss2018.1.6-17.
THE NATURAL COURSE OF CONGENITAL SPINAL DEFORMITY IN CHILDREN WITH ISOLATED VERTEBRAL BODY MALFORMATION IN THE LUMBAR SPINE

S.V. Vissarionov ${ }^{1,2}$, K.A. Kartavenko ${ }^{1}$, D.N. Kokushin ${ }^{1}$

${ }^{1}$ The Turner Scientific Research Institute for Children's Orthopaedics, St. Petersburg, Russia; ${ }^{2}$ North-Western State Medical University n.a. I.I. Mechnikov, St. Petersburg, Russia

Objective. To assess the natural history of the lumbar spine deformity progression in children with isolated vertebral body malformation. Material and Methods. The natural course of spinal deformity asso ciated with disrupted lumbar vertebra formation was analyzed in 40 patients aged 1 year 7 months to 17 years 11 months who received conservative treatment and dynamic follow-up during four years. Spondylography was performed in frontal and lateral projections in lying position every 6 months. Spondylograms were used to measure the magnitude of the angle of the local scoliotic and kyphotic compo nents of deformity in the process of child's growth and the magnitude of the angle of general lumbar lordosis. Results. The study showed that two components of deformity (scoliosis and kyphosis) were characterized by a progressive and stable course. Against the back ground of local curvature in the sagittal plane, the results of the study of general lordosis were distributed according to the age norm and to a flattening relative to the norm at the time of the last observation. Conclusion. At the primary examination of a patient, the initial mag nitude of scoliotic curvature according to Cobb plays a predictive role in determining the further course of spinal deformity. When the magnitude of the primary curve is less than $30^{\circ}$, conservative therapy provides a stable course of congenital curvature, and in some chil dren, a tendency to self-correction. Children with initial magnitude of the primary scoliotic curve of more than $30^{\circ}$ have steady progression of congenital deformity in the lumbar spine of more than $6^{\circ}$ during 4 years, which is an indication for surgical treatment at an early age. Key Words: lumbar spine, sacral spine, congenital scoliosis, hemivertebra, stable congenital deformity, progressive congenital deformity.

Please cite this paper as: Vissarionov $S V$, Kartavenko KA, Kokushin DN The natural course of congenital spinal deformity in children with isolated vertebral body malformation in the lumbar spine. Hir. Pozvonoc. 2018; 15(1):6-17. In Russian.

DOI: http://dx.doi.org/10.14531/ss2018.1.6-17. 
Пороки развития позвонков, вызывающие тяжелую и грубую деформацию позвоночника, более чем в 50 \% наблюдений локализуются в зоне грудопоясничного перехода и в поясничном отделе позвоночника [1]. Полупозвонки поясничной и пояснично-крестцовой локализации некоторые авторы относят к наиболее тяжелой врожденной патологии позвоночного столба, так как отсутствует возможность компенсации в нижележащих отделах и происходит грубое нарушение биомеханики в системе «позвоночник - таз» $[8,10]$.

Характер течения врожденной деформации позвоночника у детей с нарушением формирования позвонков определяется исходной величиной основной дуги искривления, уровнем локализации аномального позвонка, а также типом порока (сколиозогенный, кифозогенный, кифосколиозогенный).

В отечественной литературе оценка характера течения врожденной деформации позвоночника у детей раннего возраста представлена немногочисленными исследованиями $[2,12]$, согласно которым негативное влияние клиновидных позвонков и полупозвонков на течение врожденного искривления позвоночника нарастает с увеличением их количества на выпуклой стороне деформации. Прогностические критерии развития и прогрессирования врожденной деформации позвоночника обусловлены показателями активности аномальных позвонков, которые характеризуются сверхкомплектными пластинками роста в зоне костного поражения на стороне деформации, что определяется наличием или отсутствием конкресценции аномальных позвонков с соседними позвоночно-двигательными сегментами [3, 4]. Согласно данным зарубежных исследователей $[6,7$, 9], 25 \% сколиотических дуг, вызванных полупозвонком, не имеют отрицательной динамики, $25 \%$ - прогрессируют медленно, 50 \% - имеют бурное прогрессирование врожденного искривления позвоночника, которое требует хирургического лечения.
Однако авторы не выделяют течение изолированного нарушения формирования, а приводят данные наблюдений, рассматривая нарушение формирования позвонка как раздельно, так и совместно с нарушением сегментации позвонков.

Известные в настоящее время индексы прогнозирования темпов прогрессирования врожденной деформации, такие как индекс активности полупозвонка (Иа) и индекс прогрессирования деформации (Ип), предложенные Э.В. Ульрихом, а также коэффициент суммарной дисплазии (Ксд), предложенный С.А. Михайловым, имеют только исторический интерес и не используются в практическом аспекте. Вероятными признаками возможного бурного прогрессирования для сколиозогенных нарушений формирования позвонка, согласно этим данным, являются односторонние множественные полупозвонки, лежащие на вершине дуги (100 \%), исходная величина дуги более $50^{\circ}(100 \%)$, индекс прогрессирования более 0,95 (80 \%), патологическая ротация II ст. и выше (80 \%), альтернирующие позвонки, удаленные друг от друга более чем на 3 сегмента (75\%), исходная величина дуги от 30 до 50 $0^{\circ}$ (70 \%), индекс активности полупозвонка более 2,35 (70 \%) [5]. Однако, согласно практическим результатам, такая вероятность прогрессирования не всегда соответствует действительности, кроме того, расчет некоторых показателей требует многолетней динамической оценки ренттеновских снимков, что нередко приводит к формированию грубых и ригидных деформаций позвоночника у пациентов уже в дошкольном возрасте.

Цель исследования - оценка характера естественного течения деформации поясничного отдела позвоночника у детей с изолированным нарушением формирования позвонка.

\section{Материал и методы}

Под наблюдением находились 40 детей в возрасте от 1 года 7 мес. до 17 лет 11 мес. с изолированным нарушением формирования позвонка в поясничном отделе, получавших консервативное лечение и динамическое наблюдение на протяжении четырех лет. Консервативная терапия включала в себя лечебную физкультуру, направленную на укрепление мышц спины, плечевого пояса и мышц брюшного пресса, стимулирующий массаж спины, физиотерапевтическое лечение, плавание и водные процедуры. Лечебную физкультуру (статические и динамические упражнения) назначали детям старше трех лет, рекомендовали выполнять ее в игровой форме ежедневно, не менее 30-40 мин в день. Массаж спины проводили курсами 2-3 раза в год по 15 сеансов. В качестве водных процедур детям старше пяти лет рекомендовали симметричные стили плавания (брасс, баттерфляй).

Спондилографию выполняли в прямой и боковой проекциях с уровня $\mathrm{C}_{7}$ до $S_{2}$ позвонка в положении пациента лежа. По полученным спондилограммам оценивали следующие параметры:

- величину угла локального сколиотического и кифотического компонентов деформации в процессе роста ребенка, измеренного по Cobb, кратность 1 раз в 6 мес.;

- величину угла общего поясничного лордоза;

- положение таза во фронтальной плоскости;

Общий лордоз оценивали величиной угла, образованного пересечением перпендикуляров, проведенных к линиям, проходящим по краниальной замыкательной пластинке $\mathrm{L}_{1}$ позвонка и краниальной пластинке $\mathrm{S}_{1}$ позвонка. При расположении аномального позвонка в положении $\mathrm{L}_{1}$ верхнюю линию проводили вдоль краниальной пластинки нижнего грудного позвонка. При локализации полупозвонка в области поясничнокрестцового перехода нижнюю линию измерения проводили по краниальной замыкательной пластинке крестцового позвонка. Полученные результаты оценивали при помощи значений, предложенных Shefi et al. [11] (рис. 1). 


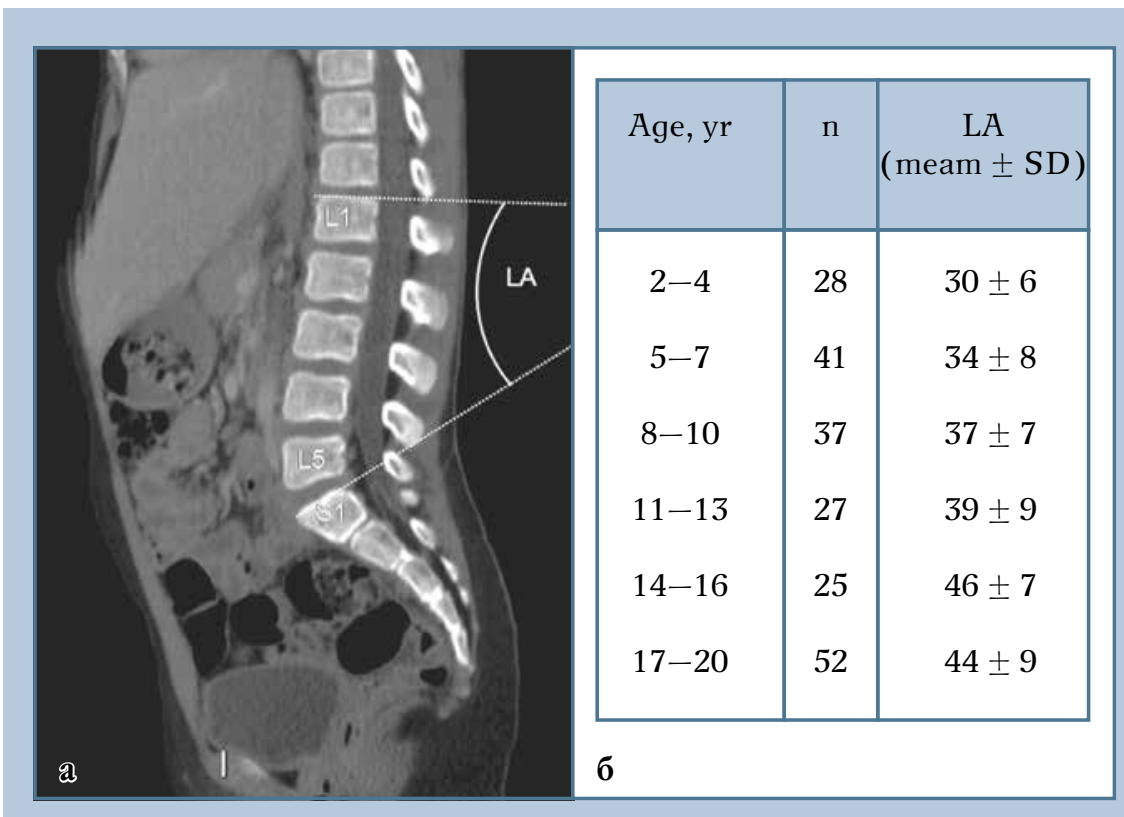

Рис. 1

Метод измерения общего поясничного лордоза (а) и нормы поясничного лордоза у детей от 2 до 20 лет (б), где age - возрастные группы по годам, $\mathrm{n}$ - количество пациентов, LA - величина поясничного лордоза по Cobb co средним отклонением

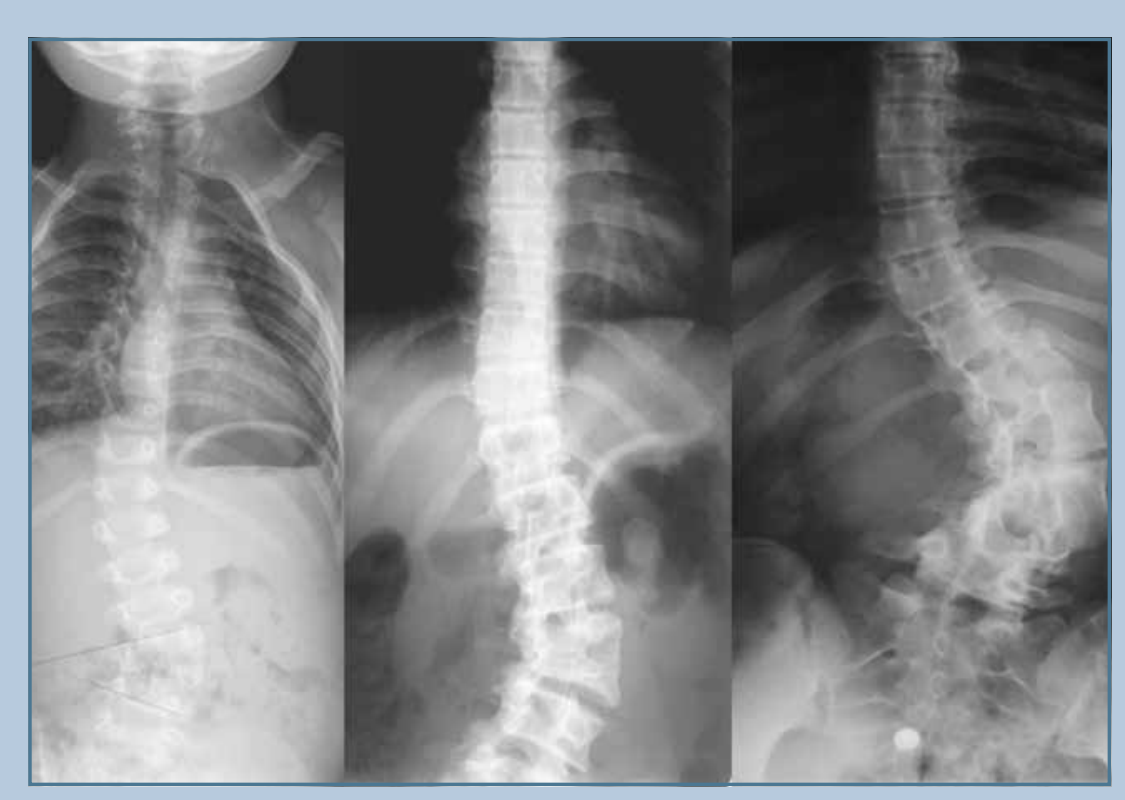

Рис. 2

Рентгенограммы позвоночника пациента Л. (№ 7) в прямой проекции, возраст от 6 лет 4 мес. до 10 лет 2 мес., с прогрессирующим сколиозом при заднебоковом левостороннем сверхкомплектном полусегментированном полупозвонке $\mathrm{L}_{4}$, конкресцированном с $\mathrm{L}_{3}$ позвонком

\section{Результаты}

Согласно результатам исследования и динамического наблюдения 2 компонента деформации (сколиоз, кифоз) характеризовались прогрессирующим и стабильным течением. На фоне локального искривления в сагиттальной плоскости результаты спондилометрического исследования общего лордоза распределялись в соответствии с величиной возрастной нормы, а также с уплощением относительно нормы на момент последнего наблюдения.

Сколиотическая дуга деформации прогрессировала у 14 (35\%) детей от 31 до $60^{\circ}$ (табл. 1).

Возраст детей с прогрессирующим сколиотическим компонентом деформации в период наблюдения - от 2,7 до 17,1 года; 8 (57 \%) полупозвонков локализовались в области грудопоясничного перехода, 6 (43\%) - на поясничном уровне. Прогрессирование сколиотического компонента деформации отмечалось в основном у пациентов с заднебоковым полупозвонком (86 \%), по сравнению с детьми с боковым полупозвонком (14 \%). Сверхкомплектные полупозвонки (57 \%) приводили к прогрессированию сколиотического компонента деформации в 57 \% наблюдений, комплектные в $43 \%$. Преимущественно к прогрессированию основной сколиотической дуги деформации приводили сегментированные полупозвонки (86 \%), по сравнению с полусегментированными (14\%).

В 2009 г. величина сколиотической дуги искривления составляла $36^{\circ}$ по Cobb, в 2011 г. - 45을 а в 2013 г. $55^{\circ}$ (рис. 2). Таким образом, за 4 года наблюдения сколиотическая деформация увеличилась практически на $20^{\circ}$.

Стабильная сколиотическая дуга наблюдалась у 26 (65 \%) пациентов. Возраст детей в период динамического наблюдения - от 3,7 до 13,9 года; 12 (46 \%) полупозвонков локализовались в области грудопоясничого перехода, 10 (38 \%) - на поясничном уровне, 4 (16\%) - в области пояснично-крестцового перехода. 


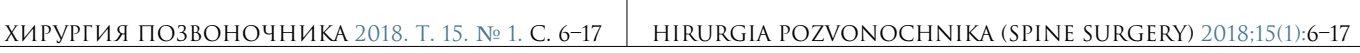

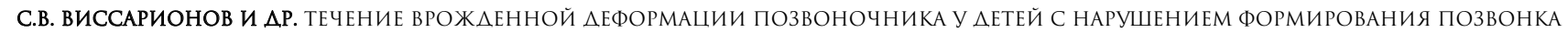
S.V. VISSARIONOV ET AL. COURSE OF CONGENITAL SPINAL DEFORMITY IN CHILDREN WITH ISOLATED VERTEBRAL BODY MALFORMATION

Таблица 1

Величина локального сколиотического компонента деформации у пациентов с изолированным нарушением формирования позвоночника

\begin{tabular}{|c|c|c|c|c|c|c|c|}
\hline \multirow[t]{2}{*}{ Пациенты } & \multirow{2}{*}{$\begin{array}{c}\text { Возраст на момент } \\
\text { наблюдения, лет }\end{array}$} & \multicolumn{6}{|c|}{ Величина сколиоза, град. } \\
\hline & & при рентгенологической & через & через & через & через & через \\
\hline
\end{tabular}

\begin{tabular}{|c|c|c|c|c|c|c|c|}
\hline $1-\tilde{n}^{*}$ & $13,5-17,4$ & 53 & 54 & 55 & 57 & 59 & 60 \\
\hline 2 -й & $5,1-9,3$ & 14 & 14 & 14 & 14 & 14 & 14 \\
\hline 3-й & $9,3-13,4$ & 22 & 22 & 21 & 21 & 20 & 20 \\
\hline 4- $\check{n}^{*}$ & $1,7-5,7$ & 33 & 33 & 34 & 36 & 38 & 40 \\
\hline 5-и́ & $4,7-8,5$ & 26 & 22 & 23 & 23 & 22 & 23 \\
\hline 6-й & $6,6-10,7$ & 15 & 14 & 14 & 14 & 14 & 14 \\
\hline 7-й & $6,4-10,2$ & 36 & 42 & 44 & 45 & 46 & 55 \\
\hline 8 -й & $4,1-8,2$ & 25 & 26 & 26 & 26 & 26 & 26 \\
\hline $9-\check{n}^{*}$ & $13,7-17,1$ & 54 & 58 & 54 & 55 & 57 & 59 \\
\hline $10-\check{n}^{*}$ & $3,8-7,6$ & 33 & 35 & 35 & 36 & 37 & 40 \\
\hline $11-\check{n}$ & $3,1-7,7$ & 27 & 22 & 22 & 22 & 22 & 22 \\
\hline $12--^{\prime \prime}$ & $10,6-14,8$ & 42 & 53 & 53 & 54 & 54 & 55 \\
\hline 13-й & $9,0-13,2$ & 24 & 25 & 25 & 24 & 23 & 23 \\
\hline 14-й & $5,1-9,1$ & 21 & 19 & 16 & 15 & 16 & 15 \\
\hline 15-й & $9,9-13,5$ & 28 & 28 & 28 & 28 & 28 & 28 \\
\hline 16-й & $4,4-8,7$ & 16 & 17 & 17 & 17 & 18 & 17 \\
\hline 17-й & $6,5-10,2$ & 26 & 21 & 21 & 21 & 22 & 22 \\
\hline $18-$ n̆ $^{*}$ & $12,1-16,8$ & 44 & 51 & 50 & 50 & 51 & 52 \\
\hline 19-и́ & $7,8-11,6$ & 27 & 27 & 28 & 28 & 28 & 28 \\
\hline $20-и ̆$ & $8,3-12,3$ & 23 & 23 & 23 & 21 & 23 & 24 \\
\hline $21-\check{\mathfrak{n}}^{*}$ & $3,6-7,8$ & 33 & 33 & 36 & 37 & 39 & 38 \\
\hline $22-$ й & $6,2-10,5$ & 17 & 17 & 20 & 21 & 20 & 20 \\
\hline 23-й & $4,2-8,1$ & 24 & 25 & 26 & 26 & 27 & 27 \\
\hline 24-й & $5,1-9,1$ & 19 & 21 & 21 & 20 & 20 & 22 \\
\hline 25-й & $7,3-11,3$ & 21 & 22 & 24 & 24 & 24 & 25 \\
\hline $26-и ̆$ & $6,7-10,6$ & 13 & 13 & 13 & 14 & 15 & 15 \\
\hline $27-и ̆$ & $5,3-9,1$ & 11 & 12 & 12 & 12 & 13 & 14 \\
\hline $28-\check{n}^{*}$ & $10,8-14,6$ & 46 & 50 & 52 & 52 & 55 & 56 \\
\hline $29-\check{n}^{*}$ & $13,1-17,7$ & 43 & 49 & 54 & 56 & 55 & 56 \\
\hline $30-$ й & $9,4-13,3$ & 29 & 28 & 28 & 29 & 29 & 29 \\
\hline 31-й & $3,7-7,1$ & 25 & 25 & 23 & 24 & 26 & 26 \\
\hline 32-й & $7,6-11,8$ & 24 & 24 & 24 & 25 & 26 & 26 \\
\hline 33-й & $9,2-13,0$ & 22 & 23 & 24 & 23 & 23 & 24 \\
\hline $34-\check{n ̆}^{*}$ & $13,4-17,5$ & 48 & 50 & 53 & 55 & 57 & 59 \\
\hline 35 -й & $4,7-8,4$ & 27 & 28 & 28 & 28 & 27 & 28 \\
\hline $36-\check{n}^{*}$ & $6,2-10,4$ & 35 & 37 & 40 & 43 & 44 & 46 \\
\hline $37-и ̆$ & $9,5-13,9$ & 24 & 24 & 23 & 24 & 25 & 26 \\
\hline $38-\check{n}^{*}$ & $12,8-16,1$ & 48 & 48 & 51 & 52 & 55 & 56 \\
\hline $39-\bar{n}$ & $4,5-8,7$ & 25 & 26 & 26 & 26 & 25 & 24 \\
\hline $40-\tilde{n}^{*}$ & $2,7-6,7$ & 33 & 37 & 37 & 35 & 36 & 39 \\
\hline
\end{tabular}

При стабильном сколиотическом компоненте деформации заднебоковые полупозвонки представлены в 16 (61\%), наблюдениях, боковые в 10 (39\%). Отсутствие прогрессиро- вания сколиотического компонента деформации отмечалось при сверхкомплектных полупозвонках (92\%). Преимущественно при отсутствии прогрессирования сколиотической дуги искривления встречались полусегментированные полупозвонки, представленные в 16 (62 \%) наблюдениях, сегментированные полупо- 


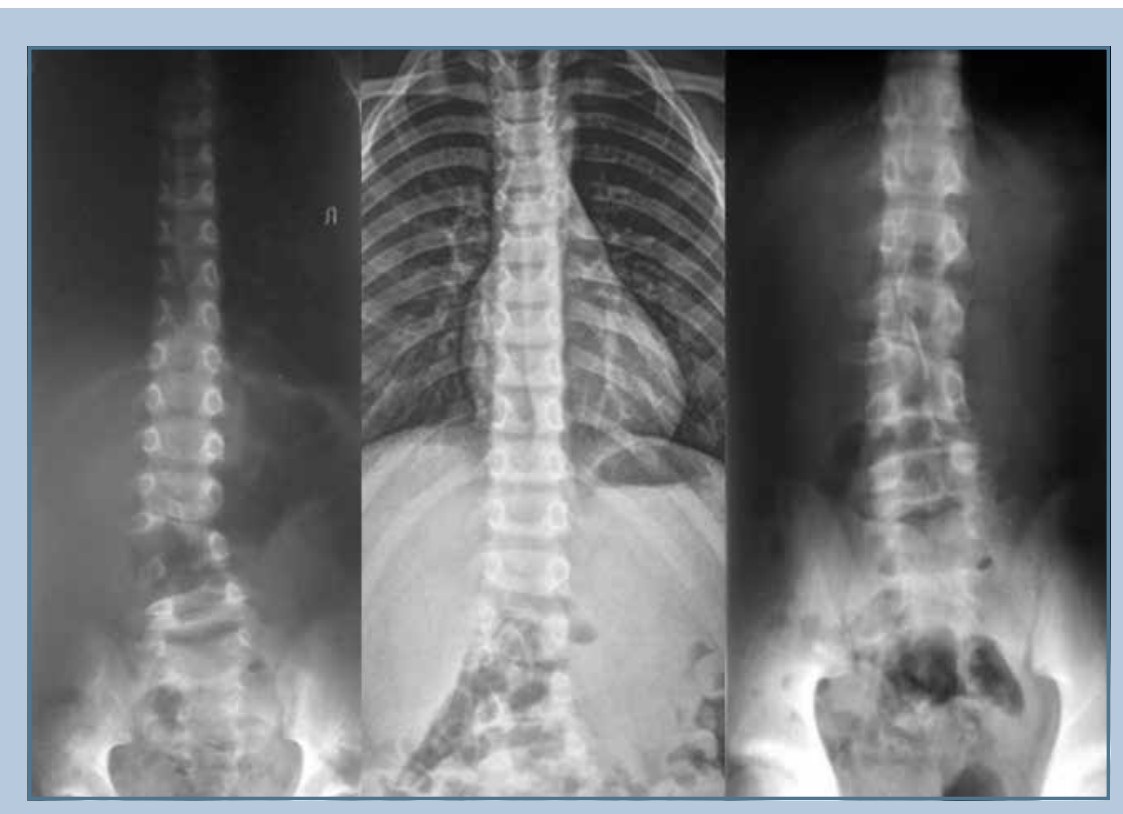

Pис. 3

Рентгенограммы позвоночника пациента 3. (№ 3) в прямой проекции, возраст от 9 лет 3 мес. до 13 лет 4 мес., со стабильным сколиозом при заднебоковом правостороннем сверхкомплектном полусегментированном полупозвонке $\mathrm{L}_{4}$, конкресцированном с $\mathrm{L}_{5}$ позвонком

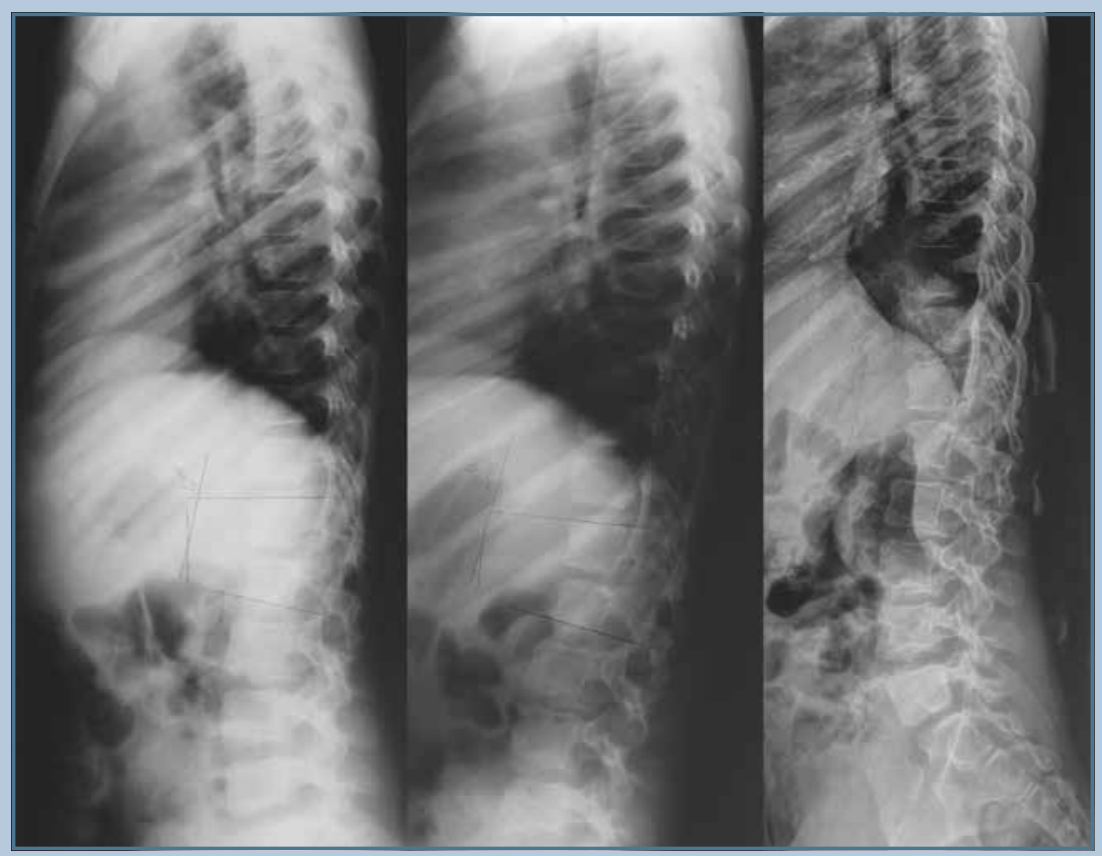

Рис. 4

Рентгенограммы позвоночника пациента М. (№ 20) в боковой проекции, возраст от 8 лет 3 мес. до 12 лет 3 мес., с прогрессирующим кифозом при заднебоковом правостороннем сверхкомплектном сегментированном полупозвонке $\mathrm{L}_{2}$ звонки отмечены у 8 (30 \%) пациентов, несегментированные - у 2 (8\%).

В 2011 г. величина сколиотического компонента деформации составила $22^{\circ}$ по Cobb, в 2013 г. $-21^{\circ}$, а в 2015 г. - $20^{\circ}$ (рис. 3). Таким образом, за 4 года динамического наблюдения отмечено отсутствие прогрессирования сколиотического компонента основной дуги искривления.

Локальный патологический кифоз прогрессировал от 8 до $60^{\circ}$ у 14 (35\%) детей (табл. 2).

Возраст детей с прогрессирующим течением локального кифоза в период наблюдения - от 4,1 до 17,1 года. Полупозвонки локализовались в грудопоясничной области - 10 (71\%) и в поясничной - 4 (29\%). По варианту нарушения формирования все 14 (100 \%) полупозвонков были заднебоковыми. Сверхкомплектные полупозвонки приводили к прогрессированию кифотического компонента деформации в 57 \% наблюдений (8 полупозвонков), в 43 \% (6 полупозвонков) аномалии представлены комплектными полупозвонками. Преимущественно в увеличении деформации в сагиттальной плоскости участвовали полусегментированные полупозвонки - у 10 (71 \%) пациентов, сегментированные - у 4 (29\%).

В 2009 г. величина локального кифоза составила $12^{\circ}$ по Cobb, в 2011 г. - $18^{\circ}$, в 2013 г. $-21^{\circ}$ (рис. 4). Таким образом, за 4 года наблюдения деформация увеличилась на $9{ }^{\circ}$.

Стабильный локальный патологический кифоз наблюдался у 26 (65 \%) детей, возраст которых в период наблюдения был от 1,8 до 16,1 года. Локализация полупозвонков по областям распределилась следующим образом: 10 (38 \%) полупозвонков в области грудопоясничного перехода, 12 (46 \%) - на поясничном уровне, 4 (16 \%) - на пояснично-крестцовом. При стабильном локальном кифотическом компоненте деформации заднебоковые полупозвонки представлены в 14 (54 \%) наблюдениях, боковые в 12 (46\%). Преимущественно стабильный кифотический компонент наблюдался при сверхкомплектном 
полупозвонке (92 \%), комплектные полупозвонки участвовали в формировании стабильного кифотического компонента в 2 (8 \%) наблюдениях. При отсутствии прогрессирования кифотического компонента сегментированные полупозвонки представлены в 16 (61,5\%) наблюдениях, полусегментированные - в 8 (30,5\%), несегментированные - в 2 (8,0 \%).

В 2010 г. величина локального кифоза составила $2^{\circ}$ по Cobb, в 2012 г. - 20, такая же величина сохранилась в 2014 г. (рис. 5). Таким образом, за 4 года в процессе роста и развития ребенка величина локального кифотического компонента деформации на уровне аномального позвонка оставалась стабильной.

Совместное прогрессирование сколиотического и кифотического компонентов деформации отмечалось у 6 (15\%) детей; совместное стабильное течение двух компонентов деформации (сколиоза и кифоза) у $18(45 \%)$.

На фоне имеющегося локального искривления результаты спондилометрического исследования общего лордоза распределились в соответ- ствии с величиной возрастной нормы, а также с уплощением относительно нормы на момент последнего наблюдения. Уплощение общего лордоза соответственно возрасту выявлено у 20 (50 \%) детей и составило от -30 до $-14^{\circ}$ (табл. 3).

Характеристика полупозвонков, приводящих к уплощению общего лордоза, представлена в табл. 4.

Величина общего поясничного лордоза соответствовала физиологической возрастной норме у 20 (50 \%) детей (табл. 5).

В результате статистического анализа величины основной сколиотической дуги при первом обследовании распределились по логарифмически нормальному закону со следующими параметрами плотности: среднее - 3,4, стандартное отклонение - 0,28.

Значения локального патологического кифоза в момент рентгенологического выявления порока были распределены по экспоненциальному закону с параметром $\theta=0,06$, критерий хи-квадрат - 0,38. При оценке анализа естественного течения сколиоза за 4 года выявлена связь с исходной величиной сколиотической дуги,

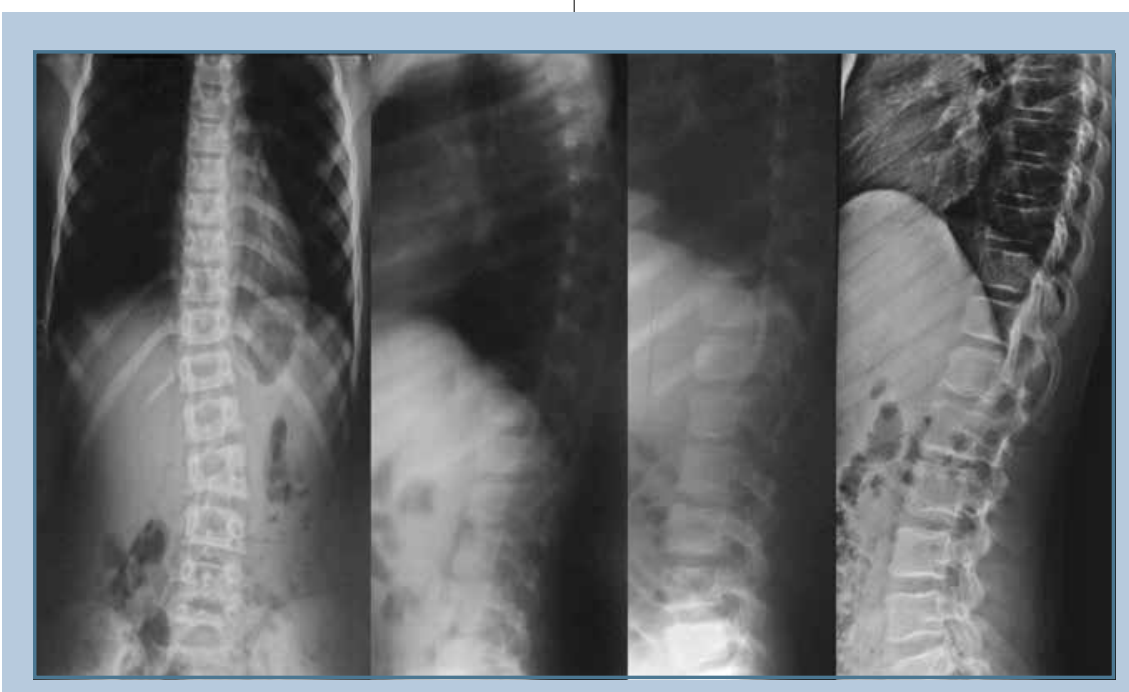

Рис. 5

Ренттенограммы позвоночника пациента С. (№ 14) в прямой и боковой проекциях, возраст от 5 лет 10 мес. до 9 лет 11 мес., со стабильным кифозом при заднебоковом левостороннем сверхкомплектном полусегментированном полупозвонке $\mathrm{L}_{3}$, конкресцированном с $\mathrm{L}_{2}$ позвонком хорошо иллюстрируемая диаграммой рассеяния этих двух признаков (рис. 6).

На диаграмме исследуемые данные детей визуально расходятся, практически формируя две категории: категория 1 - исходная величина сколиотической дуги до $30^{\circ}$ включительно, категория 2 - величина исходного локального сколиоза превышает $30^{\circ}$. На гистограмме прирост сколиотического компонента деформации в процессе динамического наблюдения в течение четырех лет распределился следующим образом: отрицательный прирост у 11 пациентов категории 1 (с исходной величиной сколиоза до $30^{\circ}$ ), из них у трех пациентов он составил от -7 до -9, у остальных - от -3 до -4․ Положительный прирост сколиоза был у 15 пациентов этой категории. У пациентов с исходной величиной локального сколиоза от $31^{\circ}$ во всех случаях (14 пациентов) наблюдался положительный прирост сколиоза. Проверку статистической значимости различия по направлению динамики прироста сколиотического угла за 4 года проводили с помощью точного критерия Фишера и подтвердили на уровне значимости $\mathrm{P}=0,0036$. Критерий Манна - Уитни подтвердил разницу между двумя категориями пациентов в приросте значений угла сколиоза за 4 года на уровне значимости $\mathrm{P}=0,0002$.

Установлено, что с приростом локального кифоза меньше $7^{\circ}$ в процессе динамического наблюдения величина общего лордоза приближается к показателям нормы соответственно возрастным критериям. Кроме того, выявлено, что при прогрессировании локального кифоза больше $7{ }^{\circ}$ в процессе роста и развития ребенка величина общего лордоза остается меныше возрастной нормы.

Согласно полученным результатам, у детей с прогрессированием локального кифоза менее $7^{\circ}$ в процессе динамического наблюдения значение общего лордоза увеличивается на бо́льшую величину угла по сравнению с пациентами, у которых прирост локального кифоза составлял более $7^{\circ}$. 


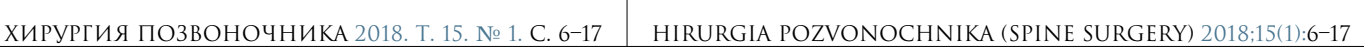

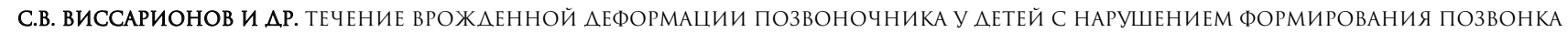
S.V. VISSARIONOV ET AL. COURSE OF CONGENITAL SPINAL DEFORMITY IN CHILDREN WITH ISOLATED VERTEBRAL BODY MALFORMATION

\begin{tabular}{|c|c|c|c|c|c|c|c|}
\hline \multirow[t]{2}{*}{ Пациенты } & \multirow{2}{*}{$\begin{array}{c}\text { Возраст на момент } \\
\text { наблюдения, лет }\end{array}$} & \multicolumn{6}{|c|}{ Величина сколиоза, град. } \\
\hline & & $\begin{array}{c}\text { при рентгенологической } \\
\text { манифестации полупозвонка }\end{array}$ & $\begin{array}{l}\text { через } \\
6 \text { мес. }\end{array}$ & $\begin{array}{l}\text { через } \\
1 \text { год }\end{array}$ & $\begin{array}{l}\text { через } \\
2 \text { года }\end{array}$ & $\begin{array}{l}\text { через } \\
3 \text { года }\end{array}$ & $\begin{aligned} \text { через } \\
4 \text { года }\end{aligned}$ \\
\hline $1-\breve{n}^{*}$ & $13,5-17,4$ & 60 & 60 & 60 & 60 & 60 & 60 \\
\hline 2 -й & $5,1-9,3$ & 8 & 9 & 11 & 13 & 14 & 15 \\
\hline 3-й & $9,3-13,4$ & 4 & 1 & 2 & 1 & 2 & 4 \\
\hline $4-\check{и}$ & $1,7-5,7$ & 2 & 4 & 3 & 4 & 5 & 4 \\
\hline 5-й & $4,7-8,5$ & 0 & 0 & 0 & 0 & 0 & 0 \\
\hline $6-\dot{n}^{*}$ & $6,6-10,7$ & 8 & 10 & 10 & 13 & 15 & 16 \\
\hline 7-й & $6,4-10,2$ & 7 & 7 & 7 & 7 & 7 & 7 \\
\hline $8-\check{n}^{*}$ & $4,1-8,2$ & 8 & 12 & 14 & 15 & 16 & 18 \\
\hline $9-\breve{n}^{*}$ & $13,7-17,1$ & 17 & 19 & 21 & 22 & 23 & 25 \\
\hline 10-й & $3,8-7,6$ & 2 & 2 & 2 & 2 & 2 & 2 \\
\hline 11-и́ & $3,1-7,7$ & 0 & 0 & 0 & 0 & 0 & 0 \\
\hline $12-\check{n}^{*}$ & $10,6-14,8$ & 10 & 18 & 20 & 22 & 23 & 24 \\
\hline 13-й & $9,0-13,2$ & 0 & 0 & 0 & 0 & 0 & 0 \\
\hline 14-й & $5,1-9,1$ & 5 & 6 & 4 & 6 & 5 & 6 \\
\hline 15-й & $9,9-13,5$ & 0 & 0 & 0 & 0 & 0 & 0 \\
\hline 16-й & $4,4-8,7$ & 11 & 11 & 10 & 10 & 11 & 11 \\
\hline 17-й & $6,5-10,2$ & 0 & 0 & 0 & 0 & 0 & 0 \\
\hline 18-й & $12,1-16,8$ & 0 & 0 & 0 & 0 & 0 & 0 \\
\hline 19-й & $7,8-11,6$ & 6 & 6 & 7 & 7 & 7 & 7 \\
\hline $20-\bar{n}^{*}$ & $8,3-12,3$ & 12 & 14 & 18 & 18 & 20 & 21 \\
\hline $21-и ̆$ & $3,6-7,8$ & 3 & 3 & 3 & 2 & 3 & 3 \\
\hline $22-и$ & $6,2-10,5$ & 0 & 0 & 0 & 0 & 1 & 1 \\
\hline $23-\breve{n}^{*}$ & $4,2-8,1$ & 8 & 9 & 12 & 14 & 15 & 17 \\
\hline 24-й & $5,1-9,1$ & 2 & 2 & 4 & 6 & 5 & 5 \\
\hline $25-\check{n}^{*}$ & $7,3-11,3$ & 13 & 13 & 15 & 17 & 19 & 19 \\
\hline $26-\check{n}^{*}$ & $6,7-10,6$ & 8 & 11 & 16 & 19 & 18 & 19 \\
\hline $27-\breve{n}^{*}$ & $5,3-9,1$ & 9 & 9 & 10 & 12 & 13 & 15 \\
\hline $28-\breve{n}^{*}$ & $10,8-14,6$ & 12 & 12 & 16 & 17 & 21 & 23 \\
\hline $29-\breve{n}^{*}$ & $13,1-17,7$ & 16 & 19 & 22 & 23 & 23 & 24 \\
\hline 30-й & $9,4-13,3$ & 0 & 0 & 0 & 1 & 0 & 0 \\
\hline $31-\bar{n}$ & $3,7-7,1$ & 0 & 0 & 2 & 3 & 3 & 3 \\
\hline 32-й & $7,6-11,8$ & 6 & 7 & 7 & 7 & 6 & 6 \\
\hline 33-й & $9,2-13,0$ & 0 & 1 & 1 & 3 & 3 & 3 \\
\hline $34-\breve{n}^{*}$ & $13,4-17,5$ & 56 & 56 & 57 & 58 & 60 & 60 \\
\hline 35-й & $4,7-8,4$ & 2 & 1 & 1 & 2 & 2 & 2 \\
\hline 36 -й & $6,2-10,4$ & 3 & 3 & 4 & 5 & 5 & 6 \\
\hline 37-и́ & $9,5-13,9$ & 0 & 1 & 1 & 1 & 2 & 2 \\
\hline $38-и$ & $12,8-16,1$ & 4 & 6 & 6 & 5 & 6 & 6 \\
\hline 39-й & $4,5-8,7$ & 0 & 0 & 0 & 0 & 0 & 0 \\
\hline 40-й & $2,7-6,7$ & 4 & 4 & 3 & 3 & 4 & 4 \\
\hline
\end{tabular}

Критерий Манна - Уитни показал статистически значимое отличие прироста локального кифоза к приросту общего лордоза на уровне значимости $\mathrm{P}=0,027$.

Полученные данные подтверждают отсутствие в исследуемых группах детей с одновременным прогрессированием локального кифоза больше $7^{\circ}$ и величиной общего лордоза, соответствующей их возрастной норме. 
ХИРУРГИЯ ПОЗВОНОЧНИКА 2018. Т. 15. № 1. С. 6-17 $\quad$ HIRURGIA POZVONOCHNIKA (SPINE SURGERY) 2018:15(1):6-17

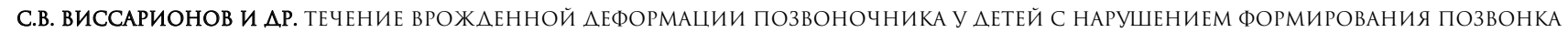
S.V. VISSARIONOV ET AL. COURSE OF CONGENITAL SPINAL DEFORMITY IN CHILDREN WITH ISOLATED VERTEBRAL BODY MALFORMATION

Таблица 3

Величина общего лордоза у пациентов с изолированным нарушением формирования позвоночника

\begin{tabular}{|c|c|c|c|c|c|c|c|c|}
\hline \multirow[t]{2}{*}{ Пациенты } & \multirow{2}{*}{$\begin{array}{c}\text { Возраст } \\
\text { на момент } \\
\text { наблюдения, } \\
\text { лет }\end{array}$} & \multicolumn{7}{|c|}{ Общий лордоз, град. } \\
\hline & & $\begin{array}{c}\text { возрастная } \\
\text { норма }\end{array}$ & $\begin{array}{c}\text { при рентгеноло- } \\
\text { гической манифе- } \\
\text { стации порока }\end{array}$ & $\begin{array}{l}\text { через } \\
6 \text { мес. }\end{array}$ & $\begin{array}{l}\text { через } \\
1 \text { год }\end{array}$ & $\begin{array}{l}\text { через } \\
2 \text { года }\end{array}$ & $\begin{array}{l}\text { через } \\
3 \text { года }\end{array}$ & $\begin{array}{l}\text { через } \\
4 \text { года }\end{array}$ \\
\hline
\end{tabular}

\begin{tabular}{|c|c|c|c|c|c|c|c|c|}
\hline $1-\dot{u}^{*}$ & $13,5-17,4$ & $-44 \pm 9$ & -14 & -14 & -14 & -14 & -14 & -14 \\
\hline 2 -й & $5,1-9,3$ & $-37 \pm 7$ & -30 & -30 & -31 & -33 & -33 & -35 \\
\hline 3-й & $9,3-13,4$ & $-39 \pm 9$ & -34 & -36 & -37 & -38 & -43 & -45 \\
\hline $4-и ́$ & $1,7-5,7$ & $-34 \pm 8$ & -40 & -40 & -41 & -41 & -42 & -42 \\
\hline 5-й ${ }^{*}$ & $4,7-8,5$ & $-37 \pm 7$ & -15 & -17 & -17 & -21 & -21 & -21 \\
\hline 6-и́ & $6,6-10,7$ & $-37 \pm 7$ & -31 & -31 & -33 & -33 & -35 & -35 \\
\hline 7- й $^{*}$ & $6,4-10,2$ & $-37 \pm 7$ & -16 & -18 & -21 & -21 & -22 & -24 \\
\hline $8-\breve{u}^{*}$ & $4,1-8,2$ & $-37 \pm 7$ & -26 & -26 & -28 & -28 & -27 & -27 \\
\hline 9-й & $13,7-17,1$ & $-44 \pm 9$ & -46 & -46 & -44 & -44 & -45 & -45 \\
\hline $10-\tilde{u}^{*}$ & $3,8-7,6$ & $-34 \pm 8$ & -22 & -22 & -23 & -23 & -24 & -24 \\
\hline 11- ń $^{*}$ & $3,1-7,7$ & $-34 \pm 8$ & -19 & -19 & -20 & -20 & -21 & -21 \\
\hline $12-\dot{n}^{*}$ & $10,6-14,8$ & $-46 \pm 7$ & -28 & -28 & -29 & -29 & -30 & -30 \\
\hline 13-й & $9,0-13,2$ & $-39 \pm 9$ & -30 & -30 & -32 & -35 & -37 & -41 \\
\hline $14-\breve{n}^{*}$ & $5,1-9,1$ & $-37 \pm 7$ & -15 & -15 & -18 & -20 & -20 & -24 \\
\hline 15-и́ & $9,9-13,5$ & $-39 \pm 9$ & -37 & -37 & -39 & -41 & -41 & -45 \\
\hline $16-\breve{n}^{*}$ & $4,4-8,7$ & $-37 \pm 7$ & -22 & -24 & -24 & -26 & -28 & -29 \\
\hline 17-и́ & $6,5-10,2$ & $-37 \pm 7$ & -36 & -36 & -39 & -41 & -41 & -43 \\
\hline 18-й ${ }^{*}$ & $12,1-16,8$ & $-46 \pm 7$ & -16 & -20 & -24 & -25 & -26 & -28 \\
\hline 19-и́ & $7,8-11,6$ & $-39 \pm 9$ & -43 & -43 & -45 & -45 & -47 & -47 \\
\hline $20-\tilde{n}^{*}$ & $8,3-12,3$ & $-39 \pm 9$ & -14 & -18 & -20 & -24 & -24 & -26 \\
\hline $21-\check{L}^{\prime}$ & $3,6-7,8$ & $-39 \pm 9$ & -20 & -21 & -22 & -25 & -25 & -26 \\
\hline $22-и ̆$ & $6,2-10,5$ & $-39 \pm 9$ & -34 & -36 & -37 & -40 & -39 & -40 \\
\hline $23-\breve{n}^{*}$ & $4,2-8,1$ & $-44 \pm 9$ & -25 & -26 & -26 & -28 & -28 & -29 \\
\hline 24-и́ & $5,1-9,1$ & $-37 \pm 7$ & -17 & -18 & -20 & -21 & -23 & -25 \\
\hline $25-\hat{n}^{*}$ & $7,3-11,3$ & $-37 \pm 7$ & -16 & -16 & -17 & -20 & -22 & -25 \\
\hline $26-\check{и}$ & $6,7-10,6$ & $-37 \pm 7$ & -30 & -31 & -32 & -34 & -34 & -36 \\
\hline 27-й & $5,3-9,1$ & $-37 \pm 7$ & -32 & -33 & -33 & -35 & -36 & -37 \\
\hline $28-\hat{n}^{*}$ & $10,8-14,6$ & $-37 \pm 7$ & -25 & -27 & -28 & -28 & -29 & -30 \\
\hline 29-й & $13,1-17,7$ & $-44 \pm 9$ & -40 & -42 & -44 & -44 & -44 & -46 \\
\hline 30-и́ & $9,4-13,3$ & $-39 \pm 9$ & -37 & -37 & -38 & -40 & -44 & -47 \\
\hline 31- nै $^{*}$ & $3,7-7,1$ & $-34 \pm 8$ & -20 & -20 & -22 & -23 & -22 & -23 \\
\hline $32-\hat{n}^{*}$ & $7,6-11,8$ & $-46 \pm 7$ & -41 & -41 & -42 & -44 & -45 & -47 \\
\hline 33-и́ & $9,2-13,0$ & $-39 \pm 9$ & -31 & -33 & -36 & -40 & -42 & -43 \\
\hline $34-\hat{n}^{*}$ & $13,4-17,5$ & $-37 \pm 7$ & -16 & -16 & -17 & -17 & -17 & -18 \\
\hline $35-\dot{n}^{*}$ & $4,7-8,4$ & $-34 \pm 8$ & -24 & -25 & -23 & -27 & -30 & -33 \\
\hline $36-\breve{n}^{*}$ & $6,2-10,4$ & $-37 \pm 7$ & -16 & -17 & -18 & -20 & -24 & -25 \\
\hline $37-и ̆$ & $9,5-13,9$ & $-37 \pm 7$ & -30 & -30 & -34 & -36 & -40 & -42 \\
\hline $38-\hat{n}^{*}$ & $12,8-16,1$ & $-46 \pm 7$ & -16 & -17 & -18 & -20 & -22 & -25 \\
\hline 39-й & $4,5-8,7$ & $-39 \pm 9$ & -16 & -17 & -18 & -20 & -22 & -23 \\
\hline 40-и́ & $2,7-6,7$ & $-34 \pm 8$ & -42 & -45 & -45 & -48 & -48 & -47 \\
\hline
\end{tabular}

\section{Обсуждение}

При исходном значении основной локальной сколиотической дуги деформации в поясничном отделе меньше $30^{\circ}$ на фоне консервативной терапии отмечали стабильное течение искривления у 65 \% детей.
Стабильность течения врожденной деформации в процессе динамического наблюдения проявлялась сохранением изначальной величины ско- 


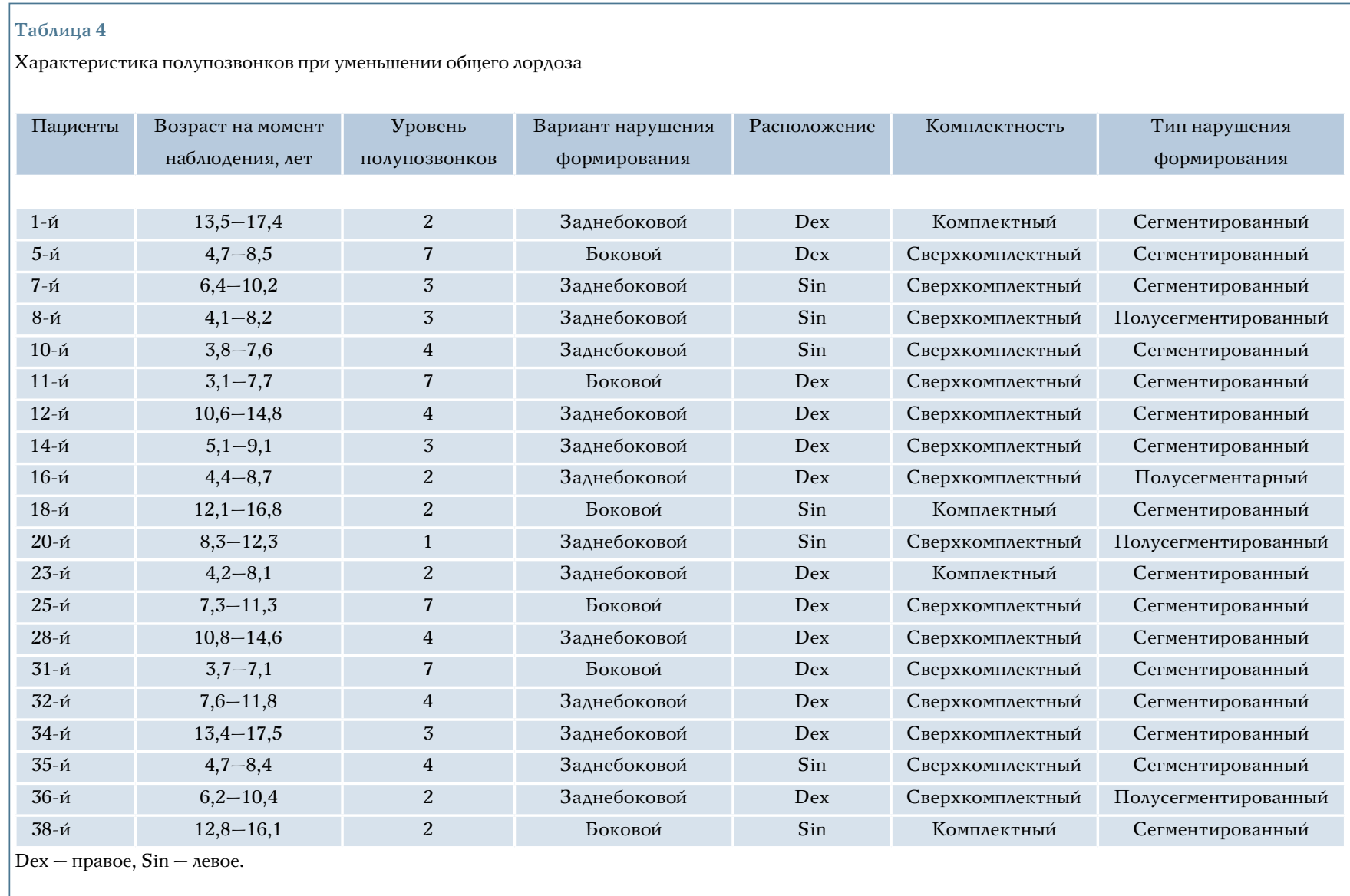

лиотического угла у 5 \% пациентов, незначительным прогрессированием локальной основной дуги, не превышающим $4^{\circ}$ за 4 года - у 37,5 \% детей, тенденцией к самокоррекции врожденного искривления в пределах 1-3 - у 22,5 \%. У всех детей с исходной величиной локальной сколиотической дуги деформации более $30^{\circ}$ отмечали неуклонное прогрессирование врожденного искривления в пределах 6-13 за 4 года динамического наблюдения.

При исходном значении основной локальной кифотической дуги деформации в поясничном отделе до $7^{\circ}$ на фоне консервативного лечения установлено стабильное течение врожденного искривления в сагиттальной плоскости. Стабильность течения проявлялась отсутствием прогрессирования деформации у 40 \% пациентов или незначительным увеличением величины локального кифоза в пределах 1-3 у 25 \% детей. У всех детей с исходной величиной локальной кифотической дуги деформации в поясничном отделе более $8^{\circ}$ отме чали неуклонное прогрессирование врожденного искривления в процессе динамического наблюдения в пределах от 6 до $14^{\circ}$.

Установлено, что в процессе динамического наблюдения при величине локального угла кифотической деформации в поясничном отделе до $7^{\circ}$ включительно значение общего лордоза, даже при исходном его уплощении, достигало возрастной нормы. У пациентов с прогрессирующим локальным кифозом более $8^{\circ}$ значение величины общего лордоза не достигало возрастной нормы и сохраняло уплощение в процессе динамического наблюдения.

Согласно данным нашего исследования, локализация аномальных полупозвонков не влияла на характер течения врожденной деформации. Наиболее частая локализация порочно развитых позвонков при стабильном течении деформации и при прогрессировании имеющегося искривления отмечена в области грудопоясничного перехода. Поясничная локализация полупозвонка представлена в меньшем соотношении к грудопоясничному расположению как при прогрессирующем, так и при стабильном течении врожденной деформации позвоночного столба. Полупозвонков, приводящих к прогрессированию сколиотической деформации и локализующихся в области пояснично-крестцового отдела, в нашем исследовании не было. Перекос таза во фронтальной плоскости наблюдался у всех детей с прогрессирующим течением врожденной деформации поясничного отдела. При прогрессировании врожденной деформации поясничного отдела позвоночника увеличивается протяженность сколиотической дуги искривления, в которую вовлекаются соседние от смежных с аномальным позвонки, а также появляется их патологическая ротация. 


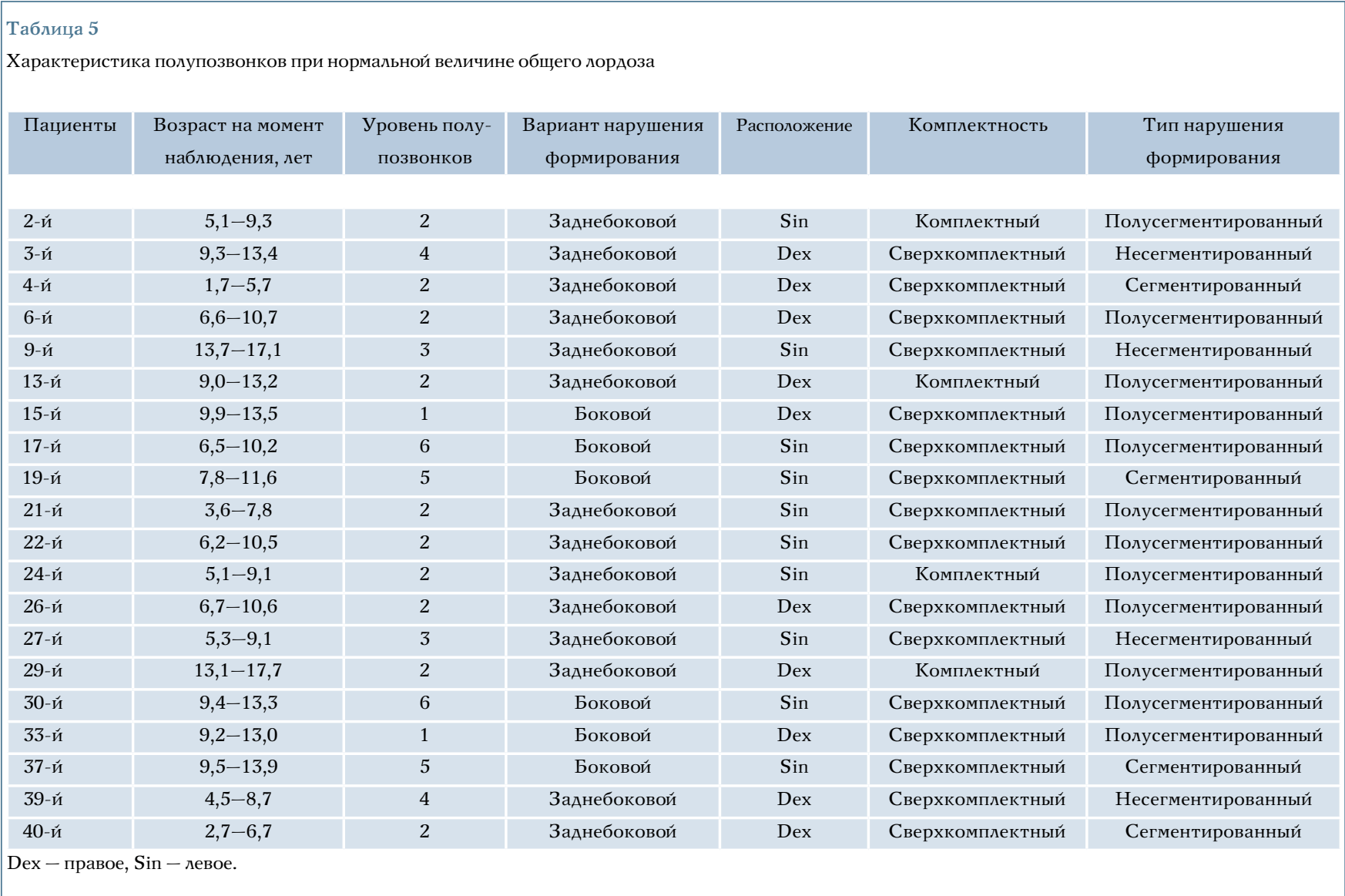

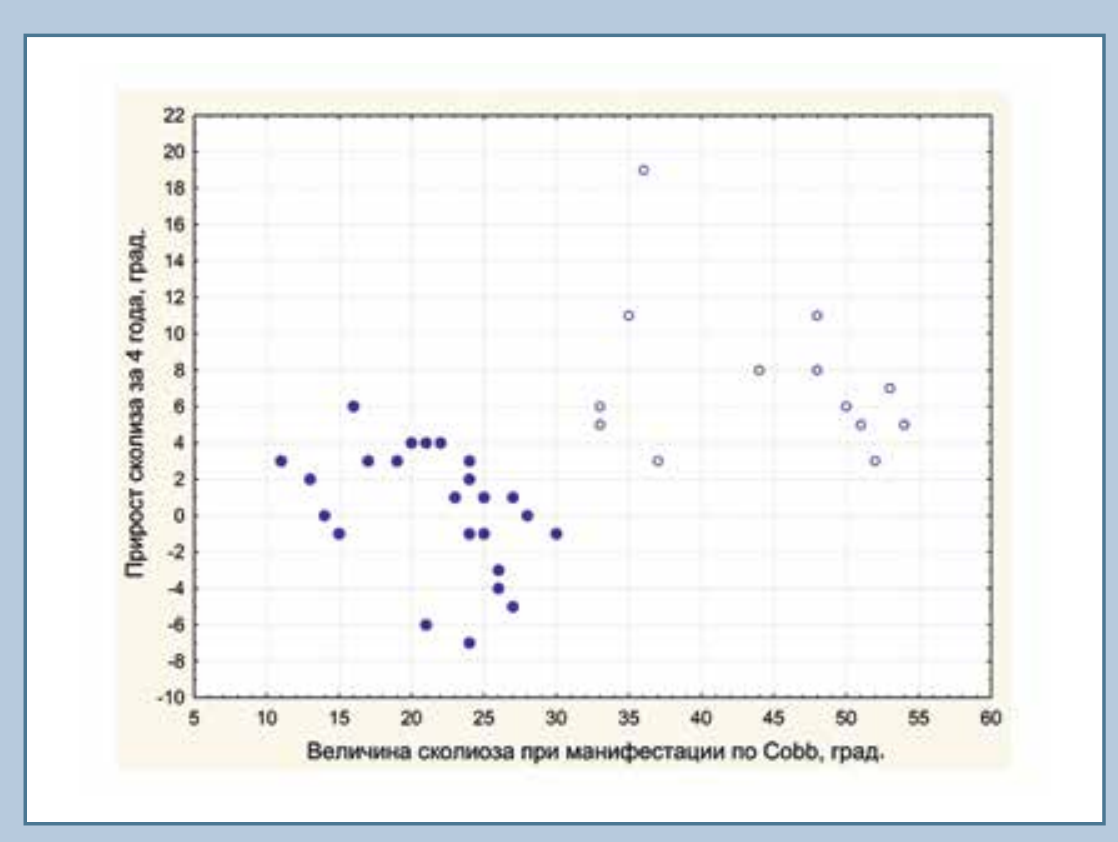

Рис. 6

Диаграмма рассеяния данных манифестации сколиотической дуги и динамики ее течения

\section{Выводы}

1. Заднебоковые полупозвонки приводят к прогрессированию всех компонентов деформации чаще, чем боковые аномальные позвонки, при которых не отмечается формирования и прогрессирования локального патологического кифоза на уровне порочного позвонка $(\mathrm{P}<0,05)$.

2. Комплектность аномального позвонка в поясничном отделе позвоночника влияет на определение характера течения врожденной деформации. Статистически установлено, что комплектные полупозвонки практически не встречаются в наблюдениях со стабильным течением врожденного искривления, а приводят к прогрессирующему течению врожденной деформации $(\mathrm{P}<0,05)$.

3. К прогрессированию основной сколиотической дуги деформации в поясничном отделе позвоночника приводят преимущественно сегментированные полупозвонки в срав- 
нении с полусегментированными. Однако в ходе динамического наблюдения установлено, что полусегментированные полупозвонки приводят к прогрессированию локальной патологической кифотической деформации поясничного отдела позвоночника, аналогично сегментированным полупозвонкам.

4. При первичном осмотре пациента прогностически ведущую роль в определении дальнейшего характеpa течения деформации позвоночника играет величина исходной сколиотической дуги искривления по Cobb. При значении основной дуги деформации меньше $30^{\circ}$ на фоне консервативной терапии отмечается стабиль- ное течение врожденного искривления, а у некоторых детей - тенденция к самокоррекции. У детей с исходной величиной основной сколиотической дуги более $30^{\circ}$ неуклонно прогрессирует врожденная деформация поясничного отдела позвоночника (больше $6^{\circ}$ за 4 года), что является показанием к хирургическому лечению уже в раннем возрасте $(\mathrm{P}=0,0036)$.

5. При исходном значении величины локального кифоза в зоне аномального позвонка меньше $7^{\circ}$ в процессе динамического наблюдения отмечается стабильное течение врожденной деформации. У таких детей величина общего лордоза в поясничном отделе на протяжении четырех лет наблюде- ния приближается к показателям нормы соответственно возрастным критериям. При исходном значении величины локального патологического кифоза более $8^{\circ}$ наблюдается прогрессирование врожденной деформации в процессе динамического наблюдения $(\mathrm{P}=0,027)$.

Работа выполнена в рамках программы Союзного государства «Разработка новых спинальных систем с использованием технологий прототипирования в хирургическом лечении детей с тяжелыми врожденными деформащиями и повреждениями позвоночника». Авторы заявляют об отсутствии конфликта интересов.

\section{Литература/References}

1. Виссарионов С.В. Хирургическое лечение сегментарной нестабильности грудного и поясничного отделов позвоночника у детей: Дис. ... д-ра мед. наук. СПб., 2008. [Vissarionov SV. Surgical treatment of segmental instability of the thoracic and lumbar spine in children. DMSc Thesis. St. Petersburg, 2008. In Russian].

2. Виссарионов С.В., Казарян И.В., Белянчиков С.М. Хирургическое лечение детей дошкольного возраста с врожденными нарушениями формирования позвонков поясничного отдела // Хирургия позвоночника. 2009. № 4. С. 44-49. [Vissarionov SV, Kazaryan IV, Belyanchikov SM. Surgical treatment of preschool children with congenital developmental disorders in the lumbar spine. Hir. Pozvonoc. 2009;(4):44-49. In Russian]. DOI: http://dx.doi.org/10.14531/ss2009.4.44-49.

3. Михайловский М.в., Фомичев Н.г. Хирургия деформаций позвоночника. Новосибирск, 2002. [Mikhaylovskiy MV, Fomichev NG. Surgery of Spinal Deformities. Novosibirsk, 2002. In Russian].

4. Михасевич Н.о., Тесаков Д.к., Тихомирова Т.Ф., Пашкевич Л.А., Ильясевич И.А., Мухля А.М. Классификация врожденных аномалий развития позвоночника // Военная медицина. 2007. № 3. С. 18-21. [Mikhasevich NO, Tesakov DK, Tikhomirova TF, Pashkevich LA, Ilyasevich IA, Mukhlya AM. Classification of congenital anomalies in the development of the spine. Military Medicine. 2007;(3):18-21. In Russian].

5. Ульрих Э.В., Мушкин А.Ю., Губин А.В. Врожденные деформации позвоночника у детей: прогноз эпидемиологии и тактика ведения // Хирургия позвоночника. 2009. № 2. C 55-61. [Ulrikh EV, Mushkin AY, Gubin AV. Congenital spine deformities in children: epidemiological prognosis and management. Hir. Pozvonoc. 2009;(2):55-61. In Russian]. DOI: http://dx.doi.org/10.14531/ss2009.2.55-61.

6. Arlet V, Odent T, Aebi M. Congenital scoliosis. Eur Spine J. 2003;12:456-463. DOI: 10.1007/s00586-003-0555-6.

7. Debnath UK, Goel V, Harshavardhana N, Webb JK. Congenital scoliosis - Quo vadis ? Indian J Orthop. 2010;44:137-147. DOI: 10.4103/0019-5413.61997.

8. Hefti F. Congenita le Skoliosen: Halbwirbelresektion-Indikationen und Techniken. Kongresstage Ortopedie, Abstracts Kongress „Kinderorthop die” 2008:18.

9. Hensinger RD. Congenital scoliosis: etiology and associations. Spine. 2009;34:17451750. DOI: 10.1097/BRS.0b013e3181abf69e.
10. King JD, Lowery GL. Results of lumbar hemivertebral excision for congenital scoliosis. Spine. 1991;16:778-782

11. Shefi S, Soudack M, Konen E, Been E. Development of the lumbar lordotic curvature in children from age 2 to 20 years. Spine. 2013;38:e602-e608. DOI: 10.1097/ BRS.0b013e31828b666b.

12. Ulrikh EV, Vissarionov SV, Mushkin AYu. Surgery for congenital vertebral abnormalities using transpedicular fixation implants in infants. Digest of Spine Surgery Journal. 2013:1-8. Electronic resource. URL: http://www.spinesurgery.ru/Digest/ Archive-DSSJ/DSSJ-2013/DSSJ-2013_82.html.

Адрес для переписки:

Кокушин Дмитрий Николаевич

196603, Россия, Санкт-Петербург, Пушкин,

ул. Парковая, 64-68, Научно-исследовательский детский ортопедический институт им. Г.И. Турнера,

partgerm@yandex.ru

Address correspondence to:

Kokushin Dmitry Nikolayevich

The Turner Scientific and Research Institute for Children's

Orthopaedics, Parkovaya str., 64-68, Pushkin,

St. Petersburg, 196603, Russia,

partgerm@yandex.ru

Статья поступила в редакцию 22.08.2017

Рецензирование пройдено 02.10.2017

Подписана в печать 12.10.2017

Received 22.08.2017

Review completed 02.10 .2017

Passed for printing 12.10.2017 
С.В. ВИССАРИОНОВ И АР. ТЕЧЕНИЕ ВРОЖАЕННОЙ АЕФОРМАЦИИ ПОЗВОНОЧНИКА У АЕТЕЙ С НАРУШЕНИЕМ ФОРМИРОВАНИЯ ПОЗВОНКА S.V. VISSARIONOV ET AL. COURSE OF CONGENITAL SPINAL DEFORMITY IN CHILDREN WITH ISOLATED VERTEBRAL BODY MALFORMATION

Сергей Валентинович Виссарионов, д-р мед. наук, проф., заместитель директора по научной и учебной работе, научный руководитель отделения патологии позвоночника и нейрохирургии, Научно-исследовательский детский ортопедический институт ил. Г.И. Турнера, ул. Парковая, 6468, 196603, Пушкин, Санкт-Петербург; проф. кафедры детской ортопедии и травматологии, Северо-Западный государственный медищиский университет им.И.И. Мечникова, Санкт-Петербург, Россия, vissarionovs@gmail.com, turner01@таil.ru;

Кирилл Александрович Картавенко, травматолог-ортопед отделения патологии позвоночника и нейрохирургии, Научно-исследовательский детский ортопедический институт им. Г.И. Турнера, ул. Парковая, 64-68, 196603, Пуикин, Санкт-Петербург, Россия, turnеr01@таil.ru;

Дмитрий Николаевич Кокуиин, канд. мед. наук, научный сотрудникотделения патологии позвоночника и нейрохирургии, Научно-исследовательский детский ортопедический институт ил. Г.И. Турнера, ул. Парковая, 64-68, 196603, Пуикин, Санкт-Петербург, Россия, partgerm@yandex.ru.

Sergei Valentinovich Vissarionov, DMSc, Prof., Deputy Director for science and academic work, Head of the Department of spine pathology and neurosurgery, The Turner Scientific Research Institute for Children's Orthopaedics, Parkovaya str., 64-68, 196603, Pushkin, St. Petersburg; Professor of the Department of Pediatric Orthopedics and Traumatology, North-Western State Medical University n.a. I.I. Mechnikov, St.Petersburg, Russia, vissarionous@gmail.com, turner01@mail.ru; Kirill Alexandrovich Kartavenko, orthopedic traumatologist in the Department of spine pathology and neurosurgery, The Turner Scientific Research Institute for Children's Orthopaedics, Parkovaya str., 64-68, 196603,Pushkin, St.Petersburg, Russia, turner01@mail.ru;

Dmitry Nikolayevich Kokushin, MD, PhD, researcher in the Department of spinal pathology and neurosurgery, The Turner Scientific Research Institute for Children's Orthopaedics, Parkovaya str., 64-68, 196603, Pushkin, St. Petersburg, Russia, partgerm@yandex.ru. 